\title{
A Fault on Mercury Revealed in 3-Dimensions
}

David L. Pegg ${ }^{1}$, David. A. Rothery ${ }^{1}$, Susan. J. Conway ${ }^{2}$, and Matthew. R. Balme ${ }^{1}$

${ }^{1}$ School of Physical Sciences, The Open University, Milton Keynes, UK (david.pegg@open.ac.uk)

${ }^{2}$ CNRS, Laboratoire de Planétologie et Géodynamique, Université de Nantes, France

Mercury's tectonic history has been dominated by global contraction as the planet cools and shrinks. Previously, fault dips have been estimated by measuring offsets to the rims of craters displaced by fault movement. Here we present the first observations of a fault surface that has been exposed in three dimensions by a volcanic vent.

Contractional tectonism is manifested through features such as lobate scarps, wrinkle ridges, and high relief ridges ${ }^{1,2}$. The stresses in Mercury's crust that generated this contractional tectonism have multiple possible origins. These include global contraction ${ }^{3}$, tidal despinning ${ }^{4}$, true polar wander ${ }^{5}$, and mantle convection ${ }^{6}$. A combination of factors probably generates many faults, but the dominant driver of contractional tectonics on Mercury is the global contraction of the planet ${ }^{7}$. This shrinking may be up to $10 \mathrm{~km}$ radially. The estimates of the actual amount of reduction in global radius recorded by Mercury's crust is $7.1 \mathrm{~km}$ if the average fault dip is $25^{\circ}$ but as little as 4.7 $\mathrm{km}$ if the average fault dip is $35^{\circ 11}$. An understanding of the dip of the faults on Mercury provides better input into these estimates and hence offers constraints on Mercury's thermal history.

In the past, only indirect means were available to calculate dips of thrust faults, by using the shortening and vertical offset of craters that straddle faults ${ }^{10,12}$. These studies derived an average dip of $25^{\circ}$. Until now, no-fault dip on Mercury has been measured by direct means.

A crater located at $147.6^{\circ} \mathrm{E},-65.6^{\circ} \mathrm{N}$ shows a small fault scarp that is visible on the side of a volcanic vent, and the trace is expressed also on the vent wall and floor. This is the first known fault surface on Mercury that is exposed in three dimensions.

While a dip determination on a single fault is not conclusive, this result suggests that the shallower dip estimates are likely more representative estimates for average fault dips on Mercury and so adds weight to the higher end of the estimates of global contraction. Unfortunately, the resolution is insufficient for us to measure displacement by means of off-set features. BepiColombo will provide further opportunities to identify and measure similar features elsewhere on Mercury, and so help constrain the planet's tectonic processes.

References: 
